\title{
Health system challenges affecting the implementation of isoniazid preventive therapy in people living with HIV in Lesotho
}

\author{
Eltony Mugomeri ${ }^{1}$, Dedré Olivier², Wilhelmiena M. van den Heever ${ }^{2}$ \\ ${ }^{1}$ Department of Pharmacy, National University of Lesotho, Roma Campus, Maseru, Lesotho \\ ${ }^{2}$ Department of Health Sciences, Central University of Technology, Bloemfontein, South Africa
}

\begin{abstract}
Introduction: The six-month course of isoniazid preventive therapy (IPT) has been demonstrated to have significant benefits in mitigating the occurrence of tuberculosis (TB), particularly in high TB burden settings. However, its implementation in Sub-Saharan countries remains subdued for obscure reasons. This study investigated the factors inhibiting IPT uptake in the sub-Saharan country of Lesotho, which has one of the highest rates of TB incidences globally.

Material and methods: Data were obtained from 46 healthcare workers, key informants at the Ministry of Health of Lesotho, and representatives of partner organizations, who were purposively selected for their roles in IPT implementation. Data were coded to identify themes, and the emerging themes were benchmarked to previous typologies for evaluating the implementation of best practices in health interventions, namely effectiveness, reach, sustainability, and adaptation. Each major theme was further linked to the World Health Organization's 'six building blocks of a health system'.

Results: Challenges affecting the implementation of IPT were as follows: ineffective TB screening due to challenged decentralization of human immunodeficiency virus (HIV)/TB services, late detection of side effects linked to weak monitoring systems, and inefficient health information systems. Further challenges in the health system included interrupted supply chains due to insufficient health system financing, while inadequate healthcare workers' education on IPT implementation was also noted.

Conclusions: These findings indicate that a wide spectrum of challenges has affected the implementation of IPT in Lesotho, and this indicates the need for 'health systems approach' to the implementation of IPT in Lesotho and other countries with similar challenges.
\end{abstract}

HIV AIDS Rev 2018; 17, 4: 299-307

DOI: https://doi.org/10.5114/hivar.2018.80263

Key words: health system challenges, isoniazid preventive therapy, people living with HIV, tuberculosis, uptake of health interventions.

\section{Introduction}

Tuberculosis (TB) is the most frequent life-threatening opportunistic disease in people living with human immunodeficiency virus (HIV). Of the 1.5 million people who died

from TB in 2013 alone, 360,000 of them were HIV-positive [1]. Yet, preventative interventions to curb the incidence of TB in this subgroup often fail to take off to scale [2]. One such intervention is isoniazid preventive therapy (IPT) recommended by World Health Organization (WHO) [3] as a pre-

Article history:

Received: 12.01.2018

Received in revised form: 26.04.2018

Accepted: 30.04.2018

Available online: 20.11.2018
International Journal of HIV-Related Problems

HIV \& AIDS

R e v i e w 
ventative therapy against TB in people living with HIV (PLHIV). A number of studies have reported the uptake of IPT in various countries and concluded that this intervention had a sluggish scale up [4-7]. However, the factors supporting its implementation are not well understood in some countries.

Sluggish scale ups of health interventions in developing countries is not uncommon and lessons can be drawn from challenges in the past [8]. McCannon et al. [9] and Buekens et al. [10] noted that sound solutions to improve scaling up of health interventions exist but their adoption is slow. The task of evaluating the implementation of health interventions, although daunting due to the spectrum of challenges involved, is important [2]. Often such evaluations lead to discovering deficiencies in the healthcare systems under investigation [11].

WHO [11] recommend increasing access to new health interventions without compromising the quality and safety of the interventions. Thus, the evaluation of implementation of new health interventions should assess the balance between access, coverage, quality, and safety through judging how well the "six building blocks" of health systems, such as service delivery, health workforce, information, medical products, financing, and leadership have been painstakingly considered in the new intervention [11]. Furthermore, the evaluation of health interventions should also include the evaluation of qualitative and quantitative evidence of best practices in local settings namely, effectiveness, reach, feasibility, sustainability, and transferability, where transferability refers to the extent to which the practice can be applied to or adapted to various contexts [12].

Getahun et al. [13], who investigated IPT from its early years of implementation, noted that barriers to IPT implementation varied from country to country, which indicates the need for more population studies of these barriers.

Lesotho is a small country, with about two million inhabitants, geographically surrounded by the Republic of South Africa [14]. The country has a mountainous terrain, with only about $10 \%$ arable land. Poverty is a problem in the country. The United Nations [15] notes that Lesotho is a poor country, with $57.1 \%$ of its population living below the official poverty line of US\$ 1.25 per day. Lesotho has the second highest estimated TB incidence in the World and a high HIV coinfection rate of about $74 \%$ of the TB cases [1].

IPT was introduced to Lesotho in 2011 [14]. Poor uptake of the drug has continued since the launch of the program [14]. This paper analyzes the health system challenges affecting the uptake of IPT in PLHIV in the high TB burden setting of Lesotho. The aim of this study is to establish, within the context of the health system of Lesotho, the compelling barriers to IPT implementation that need to be addressed.

\section{Material and methods}

\section{Study design and setting}

This qualitative study explored the health system challenges affecting IPT implementation in people living with $\mathrm{HIV}$ in Lesotho based on interviews with healthcare workers from eight district hospitals (Fig. 1), the Ministry of Health $(\mathrm{MoH})$ officials, and representatives of HIV/TB programs implementing partners, purposively sampled for their roles in the provision of HIV/TB services.

Lesotho is divided into ten administrative districts, five of which are densely populated and are considered scale up districts for HIV/TB programs. The scale up districts occupy the lowlands of the country, while the other five classified as non-scale up districts occupy the mountainous sparsely populated highlands [16].

Healthcare workers were sampled from three district hospitals in three sparsely populated (non-scale up) districts and five district hospitals from three densely inhabited (scale up) districts of the country (Fig. 1).

\section{Interview guides}

Interview guides were designed by the researchers based on the theoretical framework for developing and implementing best practices in health interventions [12] (Fig. 2). The interview guides had the following sections: 1 . Effectiveness - the extent the practice achieves the desired outcomes; 2 . Reach - the extent IPT covers the intended target population; 3 . Sustainability - the extent to which the practice can be maintained and monitored; 4. Adaptation - the extent to which the IPT intervention has been applied and adapted to the local context.

The interview guides were translated by a language specialist from English to vernacular language (Sotho), and was validated by pilot testing with ten selected healthcare workers and two key informants with experience in implementation of health interventions in Lesotho.

\section{Data collection}

Healthcare workers were interviewed first before representatives of partner organizations and the Ministry of Health $(\mathrm{MoH})$ officials to allow cross examination of the responses in the study. All healthcare workers working with IPT who were willing to participate in the study, including nurses, pharmacists, laboratory technicians, and counsellors were interviewed individually for about 30 minutes until data saturation was reached. Representatives of partner organizations were also interviewed individually, with each interview lasting for about an hour. Responses were noted down manually during the interviews and were manually transcribed to English before analysis. Transcribing was done with the assistance of a researcher with experience in qualitative coding.

\section{Data analysis}

Data were analyzed in two stages, beginning with open and axial coding to identify themes and subsequently, the emerging themes were benchmarked to previous thematic classifications according to Spencer et al. [12] typologies for evaluating the implementation of best practices in health in- 


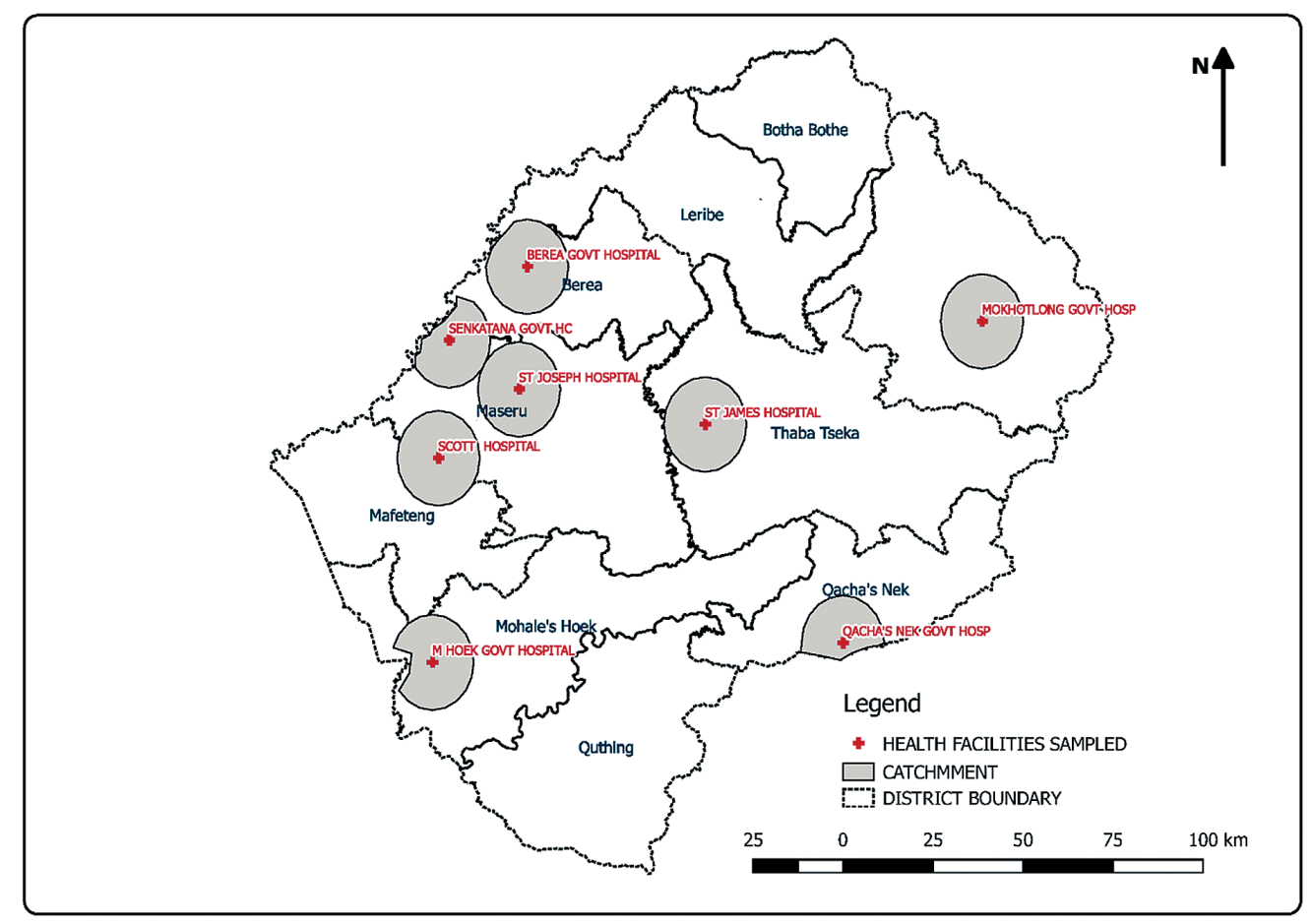

GOVT - government, HC - health center, HOSP - hospital, M. HOEK - Mohale's Hoek

Figure 1. Data collection sites. Densely populated districts included in the study were Maseru, Berea, and Mohale's Hoek, while sparsely populated districts sampled were Mokhotlong, Thaba-Tseka, and Qacha's Nek

terventions (Fig. 2). Each major theme was further classified into six subcategories, which represented the 'six building blocks of a health system' according to WHO [11] criteria for benchmarking the effectiveness of implementation of health interventions: 1. Leadership and governance; 2 . Service delivery; 3 . Supplies and products; 4 . Training and supervision; 5. Health information system; 6 . Health system financing.

To ensure reliability and consistency of data coding, interview themes were confirmed through a member checking with twenty out of 46 healthcare workers and all representatives of partner organizations and the Ministry of Health $(\mathrm{MoH})$ officials included in the study.

\section{Ethical aspects}

The study was approved by the authors institute and the Ministry of Health of Lesotho. Key informants (KIs) agreed to be interviewed through written informed consent and agreed to the use of anonymized quotations in this report. Any information that would identify the interviewees was removed from this study to protect the interviewees' anonymity.

\section{Results}

Table 1 presents the demographic information and relevant working experience of the study participants. Overall, 42 healthcare workers employed by the Government of Le-

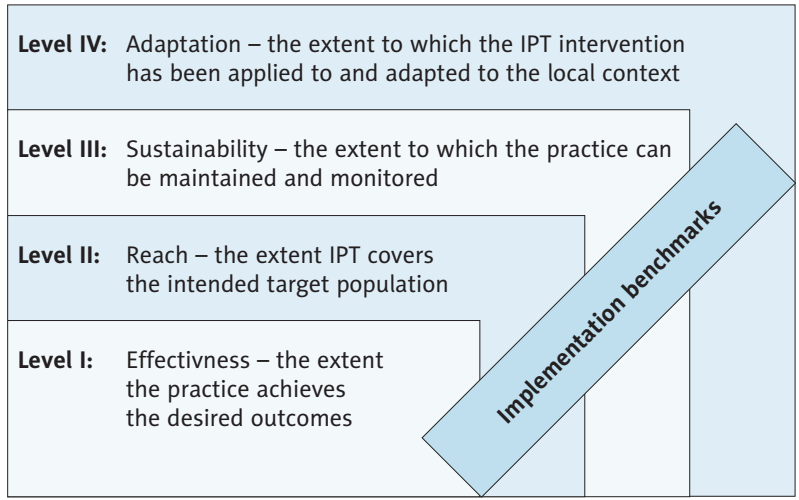

Figure 2. Benchmarks for evaluating the effectiveness of health interventions. This framework was developed based on the elements of the framework for developing and implementing best practices in health interventions by Spencer, Schooley [12]

sotho comprising nurses (33), pharmacists (5), ART counselors (2), and laboratory personnel (2) were interviewed. In addition, four nurses employed by the Elizabeth Glaser Pediatric AIDS Foundation (EGPAF), one EGPAF senior official, two Ministry of Health $(\mathrm{MoH})$ officials, and one US President's Emergency Plan for Aids Relief (PEPFAR) senior official were interviewed. 
Table 1. Demographic information and the relevant working experience of the study participants

\begin{tabular}{|c|c|c|c|c|}
\hline Participant & District & Profession & Relevant experience & Qualification \\
\hline HCW1 & Thaba Tseka & Pharmacist & 5-8 years dispensing ART/TB drugs & Honor's degree in pharmacy \\
\hline HCW2 & Thaba Tseka & Nurse & 1-7 years ART/ANC & $\begin{array}{c}\text { Diploma in general nursing and } \\
\text { midwifery }\end{array}$ \\
\hline HCW3 & Thaba Tseka & Nurse & 7- and 9-years ART & $\begin{array}{l}\text { Diploma in general nursing and } \\
\text { midwifery }\end{array}$ \\
\hline HCW8 & Thaba Tseka & Nurse & TB officer for 3 years & Diploma in general nursing \\
\hline HCW9 & Thaba Tseka & $\begin{array}{l}\text { Laboratory } \\
\text { personnel }\end{array}$ & $\begin{array}{c}5 \text { to } 7 \text { years ART laboratory } \\
\text { monitoring }\end{array}$ & $\begin{array}{c}\text { Diploma in bio-medical technology; } \\
\text { certificate in microscopy }\end{array}$ \\
\hline HCW10 & Qacha's Nek & Pharmacist & 8 years dispensing ART/TB drugs & Diploma in pharmacy technician \\
\hline HCW13 & Qacha's Nek & Nurse assistant & 6 years ART & Certificate in nurse assistance \\
\hline HCW14 & Qacha's Nek & Nurse & 4 years ART & Diploma in general nursing \\
\hline HCW15 & Qacha's Nek & Nurse & 10 years ART & $\begin{array}{l}\text { Diploma in general nursing and } \\
\text { midwifery }\end{array}$ \\
\hline HCW16 & Qacha's Nek & Nurse & 8 years ART & $\begin{array}{c}\text { Diploma in general nursing and } \\
\text { midwifery }\end{array}$ \\
\hline HCW17 & Qacha's Nek & Counsellor & $\begin{array}{l}5-6 \text { years adult ART patient } \\
\text { counselling }\end{array}$ & $\begin{array}{l}\text { Bachelor's degree in pastoral care } \\
\text { and counselling }\end{array}$ \\
\hline HCW18 & Mokhotlong & Pharmacist & 6 years dispensing ART/TB drugs & Honor's degree in pharmacy \\
\hline HCW20 & Mokhotlong & Nurse assistant & 34 years nursing experience & Certificate in nurse assistance \\
\hline HCW21 & Mokhotlong & Nurse & 3 years ART & $\begin{array}{c}\text { Diploma in general nursing and } \\
\text { midwifery }\end{array}$ \\
\hline HCW22 & Mokhotlong & Nurse & 1-year ART & Bachelor's degree in nursing \\
\hline HCW23 & Mokhotlong & Nurse & 6 years adolescent ART & $\begin{array}{c}\text { Diploma in general nursing and } \\
\text { midwifery }\end{array}$ \\
\hline HCW24 & Mokhotlong & Nurse & 7 years TB clinic & $\begin{array}{c}\text { Diploma in general nursing and } \\
\text { midwifery }\end{array}$ \\
\hline HCW25 & Mohale's Hoek & Nurse & 7 years TB clinic & Certificate in nurse assistance \\
\hline HCW26 & Mohale's Hoek & Nurse & 2 years adolescent ART & Diploma in general nursing \\
\hline HCW27 & Mohale's Hoek & Pharmacist & $\begin{array}{l}\text { (Unspecified) Years dispensing } \\
\text { ART/TB drugs }\end{array}$ & Honor's degree in pharmacy \\
\hline HCW28 & Mohale's Hoek & Nurse & 4 years ART & Diploma in general nursing \\
\hline HCW30 & Mohale's Hoek & Counsellor & 5 years adult ART patient counselling & $\begin{array}{l}\text { Bachelor's degree in pastoral care } \\
\text { and counselling }\end{array}$ \\
\hline HCW31 & Mohales' Hoek & Nurse & 4 years ART/MCH & Diploma in general nursing \\
\hline HCW32 & Mohales Hoek & Nurse & 3 years adolescent ART & Diploma in general nursing \\
\hline HCW33 & Mohales Hoek & Nurse assistant & 4 years TB clinic & Certificate in nurse assistance \\
\hline HCW34 & Maseru & Nurse & 2 years ART & Diploma in general nursing \\
\hline HCW35 & Maseru & Nurse & 4 years ART & $\begin{array}{l}\text { Diploma in general nursing and } \\
\text { midwifery }\end{array}$ \\
\hline HCW36 & Maseru & Nurse & 5 years ART & Bachelor's degree in nursing \\
\hline HCW37 & Maseru & Nurse assistant & 5 years ART & Certificate in nurse assistance \\
\hline HCW38 & Maseru & Nurse & 7 years ART/MCH & Diploma in general nursing \\
\hline HCW39 & Maseru & $\begin{array}{l}\text { Pharmacy } \\
\text { technician }\end{array}$ & $\begin{array}{c}5 \text { years assisting dispensing } \\
\text { ART/TB drugs }\end{array}$ & Diploma in pharmacy technician \\
\hline HCW40 & Maseru & Nurse assistant & 1-year TB clinic & Certificate in nurse assistance \\
\hline HCW41 & Maseru & Nurse & 2 years ART & Diploma in general nursing \\
\hline HCW42 & Maseru & Nurse & 2 years ART & Diploma in general nursing \\
\hline
\end{tabular}


Table 1. Cont.

\begin{tabular}{|c|c|c|c|c|}
\hline Participant & District & Profession & Relevant experience & Qualification \\
\hline HCW43 & Maseru & Nurse & 10 years ART & Master's degree in nursing \\
\hline HCW44 & Maseru & Nurse & 1-year adolescent ART & Bachelor's degree in nursing \\
\hline HCW45 & Maseru & Nurse & $<1$-year ART & Diploma in general nursing \\
\hline HCW46 & Maseru & Nurse & 9 years ART/TB & Master's degree in nursing \\
\hline HCW47 & Maseru & $\begin{array}{l}\text { Laboratory } \\
\text { personnel }\end{array}$ & 10 years ART laboratory monitoring & Diploma in pharmacy technician \\
\hline HCW48 & Berea & Nurse assistant & 8 years ART/MCH & Certificate in nurse assistance \\
\hline HCW49 & Berea & Nurse assistant & 11 years ART/MCH & Certificate in nurse assistance \\
\hline EGPAF1 & Berea & Nurse & $\begin{array}{c}3 \text { years EGPAF HIV/TB Clinical } \\
\text { Mentorship team }\end{array}$ & $\begin{array}{c}\text { Diploma in general nursing and } \\
\text { midwifery }\end{array}$ \\
\hline EGPAF2 & Berea & Nurse & $\begin{array}{c}5 \text { years EGPAF HIV/TB Clinical } \\
\text { Mentorship team }\end{array}$ & Bachelor's degree in nursing \\
\hline EGPAF3 & Maseru & $\begin{array}{c}\text { Quality } \\
\text { improvement } \\
\text { officer }\end{array}$ & $\begin{array}{c}5 \text { years EGPAF Quality Improvement } \\
\text { team }\end{array}$ & Bachelor's degree in nursing \\
\hline EGPAF4 & Thaba Tseka & Nurse (EGPAF) & 2 years ART/MCH nurse & Diploma in general nursing \\
\hline EGPAF5 & Maseru & Medical doctor & $\begin{array}{l}5 \text { years EGPAF senior management, } \\
\text { Lesotho }\end{array}$ & $\begin{array}{l}\text { Medical degree; Master's degree } \\
\text { in public health }\end{array}$ \\
\hline PEPFAR & Maseru & PEPFAR & PEPFAR Lesotho & $\begin{array}{l}\text { Medical degree; Master's degree } \\
\text { in public health }\end{array}$ \\
\hline WHO & Maseru & Medical doctor & $\begin{array}{l}\text { Senior management, WHO country } \\
\text { office, Lesotho }\end{array}$ & $\begin{array}{l}\text { Medical degree; Master's degree } \\
\text { in public health }\end{array}$ \\
\hline $\mathrm{MOH} 1$ & Maseru & $\begin{array}{l}\text { Public health } \\
\text { nurse }\end{array}$ & Representative at HIV/TB Directorate & $\begin{array}{l}\text { Master's degree in nursing; } \\
\text { Master's degree in public health }\end{array}$ \\
\hline $\mathrm{MOH} 2$ & Maseru & Medical doctor & Representative at HIV/TB Directorate & $\begin{array}{l}\text { Medical degree; Master's degree } \\
\text { in public health }\end{array}$ \\
\hline
\end{tabular}

EGPAF - Elizabeth Glaser Pediatric AIDS Foundation, KAPS - knowledge, attitudes, and practices, HCW - healthcare worker, PEPFAR - US President's Emergency Plan for Aids Relief, IPT - isoniazid preventive therapy, $\mathrm{MOH}$ - Ministry of Health, WHO - World Health Organization

The following sections present the themes that emerged from the interviews categorized according to the benchmarks adopted for the study.

\section{Level I: Effectiveness - the extent the practice achieves the desired outcomes}

Most of the healthcare workers were of the perception that IPT was largely effective in mitigating the occurrence of TB. They stated that cases of TB had significantly gone down in patients who had taken IPT. However, they noted that the continued occurrence of TB after taking IPT was disconcerting:

"Although the number of cases of TB among HIV patients are dwindling, TB cases continue to occur occasionally after IPT" [EGPAF1].

Major barriers to the effective implementation of IPT included ineffective monitoring and evaluation, particularly ineffective health information systems:

"The total IPT enrolment is not known because some patients are not recorded due to a shortage of registers.
Our monthly reports are sent to the $\mathrm{MOH}$ for congregate analysis but rarely get feedback" [HCW17].

On cross-interviewing, the representative of the $\mathrm{MoH}$ about the deficiency of cohort analysis of IPT indicators:

"Note that due to resource constraints, it is not feasible for $\mathrm{MOH}$ to frequently assess the effectiveness of their programs. The major goal of the $\mathrm{MOH}$ is to increase IPT uptake" [MoH1].

Despite the affirmation by healthcare workers that IPT was generally effective, most cited concerns for IPT effectiveness included poor monitoring and evaluation resulting in late detection of side effects.

"Timely detection of side effects is important. One patient reacted to concurrent cotrimoxazole and IPT two weeks after initiation of TDF/3TC/EFV" [EGPAF3].

Underreporting of monthly statistics, a point emphasized by representatives of the implementing partner organizations, was another major element affecting the efficiency of the health information system:

"Our IPT indicator system is paper-based. Inaccurate reporting and underreporting of data from healthcare centers is common" [WHO]. 
The paper-based monitoring system in the country also affected the patient referral system:

"The paper-based patient referral system in the country needs more resources including stationery and patient registers. Confirmation of patients' arrival at the referred centers is often lacking. This confounds the national monthly statistics" [PEPFAR].

Healthcare workers noted that more frequent visits of patients were required to improve monitoring. However, representatives of implementing partners had a different opinion:

"Note that the frequency of check-up dates in underresourced countries is guided by the need to balance between the amount of resources the patients have and the need for the hospital visits" [PEPFAR].

\section{Level II: Reach - the extent IPT covers the intended target population}

According to the Ministry of Health officials, IPT uptake had a sluggish start and a suboptimal trend over the years since its inception. With respect to coverage, densely inhabited districts had a lower coverage compared to the sparsely populated districts:

"Probably, about two-thirds of patients on ART have received IPT in the sparsely populated districts since 2011 compared to $50 \%$ in the densely inhabited districts" [MoH1].

In addition, IPT coverage for children, pregnant women, and spouses of PLHIV was lagging behind due to challenges in reaching these sub-groups:

"More needs to be done particularly in children. There is a considerable delay in finding the children exposed to TB" [MoH1].

"Poor uptake of HIV testing for relatives of people living with HIV particularly spouses and children is a challenge" [EGPAF3].

"HIV-positive pregnant women unaware of their HIV status are poorly covered because many of them attend ANC services as late as 36 or 38 weeks of pregnancy" [HCW48].

According to representatives of the $\mathrm{MoH}$ and partner organizations, the major barrier to IPT implementation was inadequate national planning:

"This intervention (IPT) was affected by lack of foresight at the planning stage and poor capacity to solve problems that arise" [PEPFAR].

Representatives of partner organizations noted that the government was doing its best but lacked sufficient capacity:

"The government of Lesotho is doing reasonably we; however, the MOH has no sufficient capacity to implement HIV/TB programs exclusively" [PEPFAR].

With respect to barriers inhibiting IPT reaching the target population, fear of side effects was also reiterated by healthcare workers:

"In one hospital, sporadic cases of severe side effects of liver toxicity while on IPT resulted in some ART nurses declining to prescribe IPT for months" [HCW32].
Ineffective TB screening due to an inefficient service delivery system was a major barrier to IPT uptake in many areas of the country:

"Those with presumptive signs of TB, patients declining to submit sputum for TB screening are delayed until TB is ruled out" [HCW49].

One sticking point on IPT implementation in the country is the slow scale up of IPT uptake in patients enrolled on ART before IPT was launched in 2011:

"There is a general perception that HIV patients have a low-risk of developing TB after taking ART for a long time. For this reason, IPT was mostly prescribed to new patients" [PEPFAR].

One PEPFAR representative revealed that technical challenges in reviewing the manual patient files to identify patients long on ART was also a problem.

Another theme on the delayed IPT uptake was poor staff education:

"Inadequate training is the main reason behind delayed IPT initiation. In practice, the hesitation leads to loss of patients through the cracks" [EGPAF5].

\section{Level III: Sustainability - the extent to which the practice can be maintained and monitored}

Concerning the sustainability of the intervention, additional resources were required, a seamless supply of reagents and drugs was needed:

"We often run out of stock for vitamin B6, cotrimoxazole, and dapsone (...) for adults. Vitamin B6 is supplied in small quantities" [HCW27].

$\mathrm{MoH}$ officials expressed that health system funding needs to be increased in order to improve sustainability of the HIV/TB programs including IPT. Staff shortage was another major problem:

"Staff shortages are a major challenge. Inadequate community health workers to oversee the implementation of IPT is a challenge" [MoH2].

\section{Level IV: Adaptation - the extent to which the IPT intervention has been applied and adapted to the local context}

Lack of engagement of healthcare workers responsible for implementing IPT was a major challenge inhibiting IPT uptake. Some nurses were not familiar with IPT prescription requirements for children:

"Nurses are not entirely familiar with IPT prescription and monitoring guidelines for children" [HCW49].

To address this gap and improve adaptation of IPT practice for local need, health workers needs clearer guidelines on IPT: 
"Guidelines are not clear on the duration after which IPT should be repeated. Ideally, we repeat IPT every two years in some patients" [HCW25].

Lack of clarity of guidelines was also echoed by other healthcare workers. Their concern was that there was too much room for discretional IPT prescription. This contributed to poor implementation of IPT:

"In practice, we initiate ART before IPT to unmask side effects to ARVs. However, this practice is discretional for prescribers" [EGPAF2].

\section{Discussion}

A number of challenges in the health system of Lesotho are hampering effective implementation of the IPT program. Delayed reach of IPT to some geographic locations and to children due to challenges in decentralization of HIV/TB services was a major challenge. HIV/TB services including the IPT program remain disproportionately decentralized by district despite efforts to scale up HIV/TB services in the densely populated districts of the country. Major gaps in scaling up IPT in children due to challenges hampering reaching out to children in their homes, particularly in nonscale up districts, were also a challenge. This emphasizes the need to improve decentralization of HIV/TB services to address this challenge.

Further concerns with the decentralization of critical resources for HIV/TB services were also found. The fact that the majority of healthcare workers and $75 \%$ of GeneXpert machines were located in the densely populated districts in 2014 goes beyond rational distribution of resources by population proportions and illustrates poor decentralization of HIV/TB services in the country [16]. GeneXpert machines are therefore needed to improve their distribution. Tesfaye $e t$ al. [17] also observed that there is shortage and poor distribution of GeneXpert machines in Sub-Saharan Africa and suggests that other cheaper alternatives are needed.

Late onset of antenatal care (ANC) by pregnant women, a problem commonly observed in Lesotho [18], is another challenge affecting the implementation of IPT. Tiam et al. [19] reports that at least $32 \%$ pregnant women attend ANC for the first time in the third trimester and can start IPT late. Further decentralization of healthcare services is therefore needed to improve the uptake of IPT. However, the decentralization of Lesotho's healthcare system faces many challenges including shortages of healthcare workers [20].

Ineffective TB screening and poor adherence to ART further delay IPT uptake due to the need to rule out TB before starting IPT. Ayele et al. [21] stated that lack of access to latest technologies for excluding a pre-existing TB infection prior to treatment initiation is a challenge in developing countries. This problem in Lesotho is compounded by slow uptake of GeneXpert technology. PEPFAR Lesotho [16] noted that Lesotho had a total of 28 GeneXpert machines that were not yet fully utilized due to shortages of healthcare workers, and that the problem of healthcare worker shortage, which affects TB screening resulting in slow uptake of IPT, is a typical example of health system factor interplay in Lesotho. As WHO [11] shows, dynamic interrelationships of factors in the healthcare system need to be delineated in efforts to address challenges in health systems.

Late detection of side effects to HIV/TB treatment, apparently linked to poor monitoring and evaluation of HIV services, is a challenge affecting the efficiency of the IPT intervention in Lesotho. The need to rule out side effects of ART and ensure patients are stable on ART before IPT is initiated remains a challenge. Late detection of side effects to HIV/TB treatment is also a symptom of poor staff education. It is important to note that slow implementation of critical health interventions in developing countries may be due to failure to meet minimum clinical and laboratory monitoring tests recommended by WHO [22]. To address these challenges, monitoring, evaluation, and staff development programs in the country need to be intensified [23].

Another notable theme was the need to improve patient monitoring schedules. However, to improve patient adherence to ART/IPT, evaluation studies on the merit of stricter patient monitoring schedules particularly the effectiveness of the current frequency of check-up visits, are required. PEPFAR representatives in the study noted that there is need for a balance between the amount of resources available to the patients and the need for the hospital visits.

Poor knowledge, attitudes, and practices (KAPs) were the most critical healthcare workers' factors hampering effective implementation of IPT. The need for intensive training of healthcare workers to remove the general perception that patients enrolled on ART before IPT launching have a lower risk of developing TB compared to patients enrolled on ART after IPT launching, cannot be overemphasized. Ayele et al. [21] and Noé et al. [24] emphasize the need for healthcare workers' education in high TB burden settings to improve their perceptions and knowledge of IPT practice guidelines.

Slow adaptation of IPT practice to suit the local context was another critical challenge associated with health work force factors. Healthcare workers do not seem to have a uniform approach to the implementation of IPT. Some nurses are not entirely familiar with IPT prescription and monitoring guidelines for children. The timing of IPT initiation relative to ART remains largely discretional in many hospitals with some prescribers initiating IPT six months after starting the ART and some only two weeks after ART. This indicates that there are gaps in the adaptation of IPT guidelines to the local context. Therefore, more training and supervision are needed.

The need to monitor adherence to ART before administering IPT is one critical factor hindering IPT implementation. Healthcare workers in this study delayed giving IPT to patients with poor adherence to ART until the patients were stable on ART. In addition, patients deemed too sick were also delayed. Apparently, patients considered too sick are at the greatest risk of developing TB [25]. Healthcare workers' education is therefore also needed to address this problem. 
Suboptimal monitoring of the effectiveness of the IPT program mainly due to weak monitoring system needs attention in Lesotho. Timely detection of patients getting infected by TB after IPT is necessary to inform IPT policy for improvement [26]. Without a systematic collection of data providing evidence of the effectiveness or failure of an intervention program such as IPT, it is difficult to convince healthcare workers to improve their work [27]. The lack of longitudinal analysis of data supporting the effectiveness of IPT and poor monitoring and evaluation may be a symptom of sub-optimal monitoring and evaluation system in the country. Lack of skills in healthcare workers involved in data gathering and analysis may be the root cause, while other challenges including poor implementation of electronic medical records (EMR) systems and over-reliance on paper-based monitoring systems may be nested in the problem of skills shortage in the country. Suggestions to improve monitoring systems include providing hands-on support for data collection and use at facility and national levels, utilizing web-based databases for data entry, and efficient dissemination of results [27].

The incidence of TB in Lesotho is estimated at 852/ 100,000 in the general population, making the country an ideal target for IPT because this intervention has been ear-marked for high TB burden countries [28]. The contextual underpinnings behind the slow uptake of IPT are therefore an important research problem to improve IPT uptake in the country. Clearly, opportunities to leverage the existing HIV services for better IPT uptake in Lesotho are being lost. Of note, underestimation of potential public health impact has been identified as a major cause of the delayed implementation of this intervention in Ivory Coast [29]. Healthcare workers' education is therefore needed to improve IPT implementation in the country.

The spectrum of challenges affecting the implementation of IPT is therefore wide and extents to most of the "six building blocks" of the national health system advocated by WHO [11]. The challenges can be summarized as: 1 . Ineffective $\mathrm{TB}$ screening due to challenged decentralization of HIV services and limitations in the healthcare delivery system; 2 . Late detection of side effects linked to weak monitoring systems and inefficient health information systems; 3. Interrupted supply chains due to insufficient health system financing, and 4. Poor KAPs of healthcare workers linked to inadequate healthcare workers' education.

A number of changes to the health system are therefore required to maintain and sustain the IPT program. Additional health system financing is needed to acquire supplementary healthcare workers, data clerks, stationary particularly integrated ART/IPT registers, and combined ART/ IPT dosage forms are needed. In addition, improved supply chains and additional resources are also required to sustain the program. Furthermore, physical infrastructure, laboratory upgrades in particular are needed.

Furthermore, out of the major barriers to scaling up of health interventions in developing countries outlined by Yamey [2], the barriers re-echoed in this study include: limited human resources, leadership, poor health systems capacity, and lack of engagement of local implementers. Therefore, to address the problems hampering IPT uptake in Lesotho, there is need for 'health systems approach' to the implementation of IPT, as the barriers are better addressed through a comprehensive implementation program that features contextual health system factors.

One limitation of this study was the inability to include quantitative evidence of IPT uptake to confirm perceptions of healthcare workers about IPT uptake. Further studies that triangulate quantitative evidence with qualitative findings are therefore needed in Lesotho. In addition, this study presented in isolation, without quantitative data on the effectiveness of IPT in the country leaves critical gaps for policy-making. Therefore, despite the importance of the findings of this study, the study needs to be interpreted with these limitations in mind.

\section{Conclusions}

The spectrum of the challenges curtailing the implementation of IPT in Lesotho is evidently wide. As such, these challenges call for a systems approach to addressing them. Particular themes emanating from this study that resonate with global challenges inhibiting scale up of this particular intervention in developing countries include ineffective TB screening, late detection of side effects due to weak monitoring systems, and inadequate healthcare workers' education. Clearly, these challenges need to indicate the necessity for a health systems approach to the implementation of IPT and other complex health interventions in developing countries.

\section{Funding}

This work was supported by a research grant from the authors' institute.

\section{Conflict of interest}

The authors declare no potential conflicts of interest with respect to the research, authorship, and/or publication of this article.

\section{References}

1. WHO. Global tuberculosis report 2014. World Health Organization, Geneva 2014.

2. Yamey G. What are the barriers to scaling up health interventions in low and middle income countries? A qualitative study of academic leaders in implementation science. Global Health 2012; 8: 11.

3. WHO. Interim policy on collaborative TB/HIV activities. WHO Press, Geneva 2004.

4. Adams LV, Talbot EA, Odato K, et al. Interventions to improve delivery of isoniazid preventive therapy: an overview of systematic reviews. BMC Infect Dis 2014; 14: 281.

5. Adjobimey M, Masserey E, Adjonou C, et al. Implementation of isoniazid preventive therapy in children aged under 5 years exposed to tuberculosis in Benin. Int J Tuberc Lung Dis 2016; 20: 1055-1059. 
6. Ayele HT, van Mourik MS, Bonten MJ. Predictors of adherence to isoniazid preventive therapy in HIV patients in Ethiopia: A prospective cohort study. Int J Infect Dis 2016; 45 (Suppl 1): 386.

7. Charles MK, Lindegren ML, Wester CW, et al.; International epidemiology Databases to Evaluate AIDS (IeDEA) Collaboration. Implementation of Tuberculosis Intensive Case Finding, Isoniazid Preventive Therapy, and Infection Control ("Three I's") and HIVTuberculosis Service Integration in Lower Income Countries. PLoS One 2016; 11: e0153243.

8. Lengeler C. Insecticide-treated bed nets and curtains for preventing malaria. Cochrane Database Syst Rev 2004; 2: CD000363.

9. McCannon CJ, Berwick DM, Massoud MR. The science of large -scale change in global health. JAMA 2007; 298: 1937-1939.

10. Buekens P, Keusch G, Belizan J, et al. Evidence-based global health. JAMA 2004; 291: 2639-2641.

11. WHO. Strengthening health systems to improve health outcomes: WHO's framework for action. WHO Press, Geneva 2007.

12. Spencer LM, Schooley MW, Anderson LA, et al. Seeking Best Practices: A Conceptual Framework for Planning and Improving Evidence-Based Practices. Prev Chronic Dis 2013; 10: 130186.

13. Getahun H, Granich R, Sculier D, et al. Implementation of isoniazid preventive therapy for people living with HIV worldwide: barriers and solutions. AIDS 2010; 24: S57-65.

14. GoL. National TB and Leprosy Control Strategic Plan 2013-2017. Government of Lesotho, Maseru 2013.

15. United Nations. Assessing progress in Africa toward the Millennium Development Goals. United Nations, New York 2010.

16. PEPFAR Lesotho. Lesotho Country Operational Plan (COP) 2016 Strategic Direction Summary 2016 [Online]. Available at: https:// www.pepfar.gov/documents/organization/257640.pdf (Accessed: 7 March 2017)

17. Tesfaye A, Fiseha D, Assefa D, et al. Modeling the patient and health system impacts of alternative xpert MTB/RIF algorithms for the diagnosis of pulmonary tuberculosis in Addis Ababa, Ethiopia. BMC Infect Dis 2017; 17: 318

18. Mugomeri E, Musa NK, Chatanga P. Haemoglobin response to routine iron and folate supplementation during pregnancy in an HIV-endemic rural area of Roma, Lesotho. Medical Technology SA 2016; 30: 10-14.

19. Tiam A, Machekano R, Gounder CR, et al. Preventing tuberculosis among HIV-infected pregnant women in lesotho: the case for rolling out active case finding and isoniazid preventive therapy. J Acquir Immune Defic Syndr 2014; 67: e5-e11.

20. Bemelmans M, Goux D, Baert S, et al. The uncertain future of lay counsellors: continuation of HIV services in Lesotho under pressure. Health Policy Plan 2016; 31: 592-599.

21. Ayele HT, van Mourik MS, Bonten MJ. Effect of isoniazid preventive therapy on tuberculosis or death in persons with HIV: a retrospective cohort study. BMC Infect Dis 2015; 15: 334.

22. WHO. Guidelines for intensified tuberculosis case-finding and isoniazid preventive therapy for people living with HIV in resourceconstrained settings. WHO Press, Geneva 2011.

23. Buchbinder SB, Shanks NH. Introduction to health care management. Jones \& Bartlett Publishers, 2016.

24. Noé A, Ribeiro RM, Anselmo R, et al. Knowledge, attitudes and practices regarding tuberculosis care among health workers in Southern Mozambique. BMC Pulm Med 2017; 17: 2.

25. Collins S, Juste J, Koenig S, et al. CD4 deficit and tuberculosis risk persist with delayed antiretroviral therapy: 5-year data from CIPRA HT-001. Int J Tuberc Lung Dis 2015; 19: 50-57.

26. Churchyard G, Mametja L, Mvusi L, et al. Tuberculosis control in South Africa: Successes, challenges and recommendations. S Afr Med J 2014; 104 (3 Suppl 1): 244-248.

27. Saito S, Howard AA, Chege D, et al. Monitoring quality at scale: implementing quality assurance in a diverse, multicountry HIV program. AIDS 2015; 29 Suppl 2: S129-136.
28. WHO. Global tuberculosis report 2015. WHO/HTM/TB/2015.22. WHO Press, Geneva 2015

29. Temprano ANRS 12136 Study Group; Danel C, Moh R, Gabillard D, et al. A trial of early antiretrovirals and isoniazid preventive therapy in Africa. N Engl J Med 2015; 373: 808-822. 